\title{
NEUTRAL CURRENT CROSS SECTIONS WITH POLARISED LEPTON BEAM AT ZEUS
}

\author{
S. U. NOOR \\ York University, \\ Petrie Science and Engineering Building, \\ 4700 Keele St., Toronto, Ontario, M3J 1P3, Canada
}

\begin{abstract}
Measurements of the neutral current cross sections for deep inelastic scattering in $e^{ \pm} p$ collisions with longitudinally polarised lepton beams are presented. The single differential cross section $d \sigma / d Q^{2}$ is presented for $e^{ \pm} p$. For the $e^{-} p$ data set, the double differential cross section in $Q^{2}$ and $x$ is shown and the structure function $x F_{3}$ is extracted using previously measured unpolarised $e^{+} p$ measurements. The polarised $e^{+} p$ measurements are based on an integrated luminosity of $23.8 \mathrm{pb}^{-1}$ taken by the ZEUS detector in 2004. The polarised $e^{-} p$ data has an integrated luminosity of $122 \mathrm{pb}^{-1}$ taken in 2004 and 2005. During both running periods, leptons and protons were collided at HERA with a centre-of-mass energy of $318 \mathrm{GeV}$. The Standard Model agrees well with all measurements, with the $d \sigma / d Q^{2}$ measurement showing clear evidence of parity violation.
\end{abstract}

\section{Introduction}

Deep inelastic scattering (DIS) of leptons off nucleons is a key tool to probe the structure of matter at small distance scales. The neutral current (NC) DIS interaction at HERA, $e^{ \pm} p \rightarrow e^{ \pm} X$, proceeds via the exchange of a photon or a $Z^{0}$ boson.

The kinematics of NC DIS can be defined in terms of the variables $x, y$ and $Q^{2}$. The variable $Q^{2}$ is defined to be $Q^{2}=-q^{2}=-\left(k-k^{\prime}\right)^{2}$ where $k$ and $k^{\prime}$ are the four-momenta of the incoming and scattered lepton, respectively. Bjorken $x$ is defined by $x=Q^{2} / 2 P \cdot q$ where $P$ is the fourmomentum of the incoming proton. The variables $x, y$ and $Q^{2}$ are related by $Q^{2}=s x y$, where $s=4 E_{e} E_{p}$ is the square of the lepton-proton centreof-mass energy (neglecting the masses of the incoming particles).

The Standard Model (SM) predicts that the cross section for $e^{ \pm} p \mathrm{NC}$ DIS should exhibit a dependence on the polarisation of the incoming lepton due to the parity violating nature of the weak interaction. Therefore, this polarisation effect should be most significant at high $Q^{2}$ where the $Z^{0}$ boson 
exchange becomes important.

These proceedings present the cross section measurements for $\mathrm{e}^{ \pm} \mathrm{p}$ NC DIS with longitudinally polarised lepton beams. The $\mathrm{e}^{+} \mathrm{p}$ measurements [1] are based on data with an integrated luminosity of $23.8 \mathrm{pb}^{-1}$ collected at a mean luminosity weighted polarisation of +0.32 and -0.41 with the ZEUS detector in 2004. The $\mathrm{e}^{-} \mathrm{p}$ data has an integrated luminosity of $122 \mathrm{pb}^{-1}$ with a mean luminosity weighted polarisation of +0.33 and -0.27 collected in 2004 and 2005. During both running periods HERA collided protons of energy $920 \mathrm{GeV}$ with positrons or electrons of energy $27.5 \mathrm{GeV}$, yielding collisions at a centre-of-mass energy of $318 \mathrm{GeV}$.

\section{Cross sections}

The unpolarised electroweak Born-level cross section for the $e^{ \pm} p \mathrm{NC}$ interaction can be written as

$$
\frac{d^{2} \sigma\left(e^{ \pm} p\right)}{d x d Q^{2}}=\frac{2 \pi \alpha^{2}}{x Q^{4}} H^{ \pm}
$$

where $\alpha$ is the fine-structure constant and $H^{ \pm}$is defined by

$$
H^{ \pm} \equiv Y_{+} F_{2}\left(x, Q^{2}\right) \mp Y_{-} x F_{3}\left(x, Q^{2}\right)
$$

where $Y_{ \pm} \equiv 1 \pm(1-y)^{2}$. The structure functions $F_{2}$ and $x F_{3}$ contain the sums and differences of the quark and anti-quark parton density functions (PDFs). The longitudinal structure function $F_{L}$ is ignored as it is small in the kinematic region considered.

The reduced cross section is defined as

$$
\tilde{\sigma}^{e^{ \pm} p}=\frac{x Q^{4}}{2 \pi \alpha^{2}} \frac{1}{Y_{+}} \frac{d^{2} \sigma\left(e^{ \pm} p\right)}{d x d Q^{2}}=F_{2}\left(x, Q^{2}\right) \mp \frac{Y_{-}}{Y_{+}} x F_{3}\left(x, Q^{2}\right)
$$

which is used in this analysis to extract $x F_{3}$.

The NC cross section is modified when the incoming lepton beam is longitudinally polarised. The longitudinal polarisation is defined as

$$
P_{e}=\frac{N_{R}-N_{L}}{N_{R}+N_{L}}
$$

where $N_{R}$ and $N_{L}$ are the numbers of right and left-handed leptons in the beam. By including the polarisation, the Born $e^{ \pm} p \mathrm{NC}$ cross section defined by Eq. (1) can be generalised as

$$
\frac{d^{2} \sigma\left(e^{ \pm} p\right)}{d x d Q^{2}}=\frac{2 \pi \alpha^{2}}{x Q^{4}}\left[H^{ \pm}+P_{e} H_{P_{e}}^{ \pm}\right]
$$

where $H_{P_{e}}^{ \pm}$contains the polarised structure functions. 

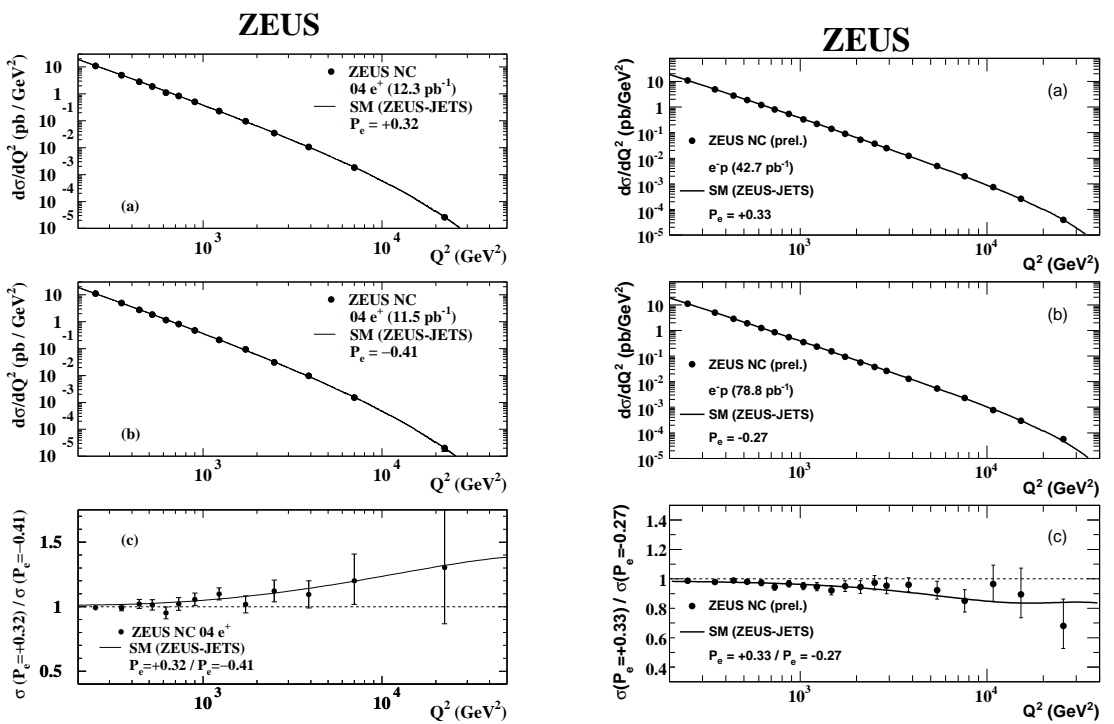

Figure 1. The $e^{+} p$ cross section $d \sigma / d Q^{2}$ is shown on the left and the $e^{-} p$ cross section $d \sigma / d Q^{2}$ is shown on the right. Both plots present $d \sigma / d Q^{2}$ for (a) positive polarisation data, (b) negative polarisation data, and (c) a ratio of the two. The curves show the predictions of the SM evaluated using the ZEUS-JETS PDFs.

\section{Results}

The cross section $d \sigma / d Q^{2}$ for $e^{ \pm} p$ NC DIS is shown in Fig. 1 for positively and negatively longitudinally polarised lepton beams. Only statistical uncertainties were considered when taking the ratio of the cross sections with the two polarisations. A clear indication of parity violation is seen as the cross section ratio deviates from unity and is well described by the SM evaluated using the ZEUS-JETS PDFs.

Figure 2 presents the reduced cross sections for unpolarised $e^{ \pm} p$ and the $x F_{3}$ measurements. The unpolarised $e^{-} p$ reduced cross sections are measured by combining the positive and negative polarisation samples, and correcting the residual polarisation of -0.06 . The reduced cross sections are compared with previously measured unpolarised $e^{+} p$ reduced cross sections taken in 1999 and 2000 [2]. A significant difference between the two data sets is seen at high $Q^{2}$ due to the $x F_{3}$ contribution. The structure function $x F_{3}$ is extracted using the unpolarised $e^{ \pm} p$ reduced cross sections and is reproduced well by the SM. 


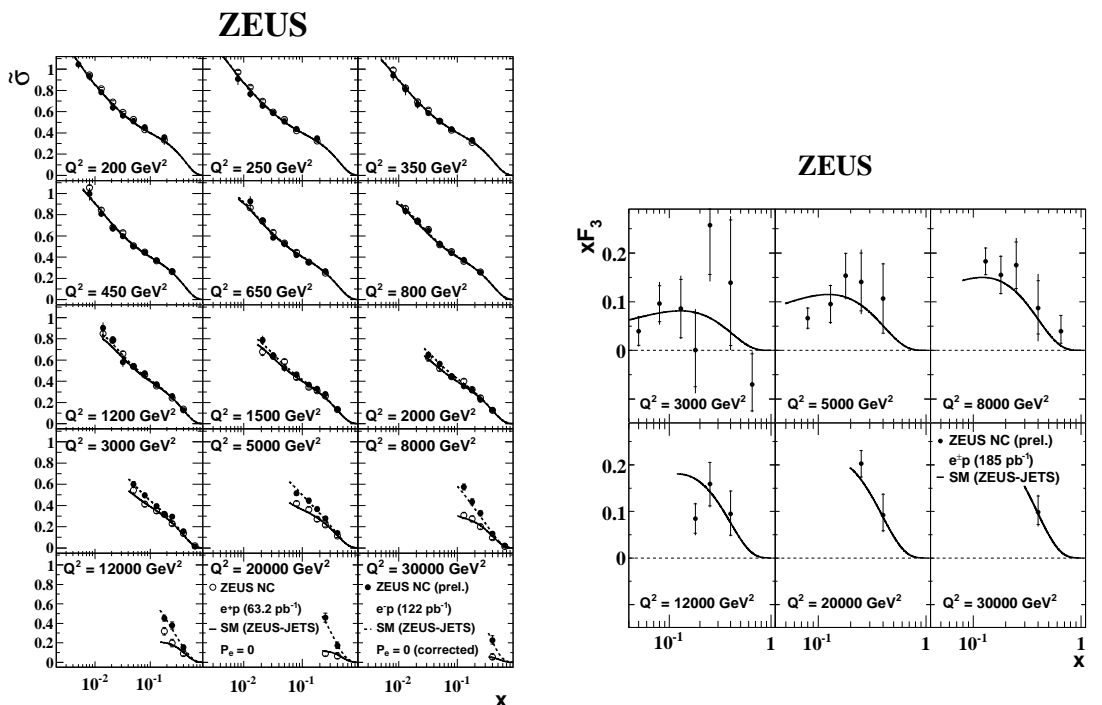

Figure 2. The $e^{ \pm} p$ unpolarised reduced cross section, $\tilde{\sigma}$, plotted as a function of $x$ in fixed $Q^{2}$ bins is shown on the left. On the right is the structure function $x F_{3}$ plotted as a function of $x$ in fixed $Q^{2}$ bins. The curves on both plots show the SM prediction evaluated using the ZEUS-JETS PDFs.

\section{Summary}

The single differential cross section $d \sigma / d Q^{2}$ is presented for $e^{ \pm} p$ NC DIS separately for positively and negatively longitudinally polarised leptons. The $e^{-} p$ reduced cross sections corrected to zero polarisation are presented and have been combined with previously measured unpolarised $e^{+} p$ reduced cross sections to extract $x F_{3}$. The $\mathrm{SM}$ predictions describe the measurements well and this is the first time at ZEUS that parity violation can clearly be seen in the $d \sigma / d Q^{2}$ measurement.

\section{References}

1. ZEUS Collab., S. Chekanov etal., Preprint hep-ex/0402026, 2006. Accepted by Phys. Lett. B

2. ZEUS Collab., S. Chekanov etal., Phys. Rev. D 70, 052001 (2004) 